\title{
Germanica
}

\section{Die Kriminalromane von Wolf Haas. Ein kritischer Dialog mit Tradition und Gegenwart}

Les romans policiers de Wolf Haas. Un dialogue critique entre tradition et présent

The detective novels by Wolf Haas. A critical dialogue with tradition and present

\section{Valentina Serra}

\section{(2) OpenEdition}

Journals

Édition électronique

URL : http://journals.openedition.org/germanica/3184

DOI : 10.4000/germanica.3184

ISSN : 2107-0784

Éditeur

Université de Lille

Édition imprimée

Date de publication : 30 septembre 2016

Pagination : 69-80

ISBN : 9782913857377

ISSN : 0984-2632

Référence électronique

Valentina Serra, «Die Kriminalromane von Wolf Haas. Ein kritischer Dialog mit Tradition und Gegenwart », Germanica [Online], 58 | 2016, Online erschienen am: 30 September 2018, abgerufen am 06 Oktober 2020. URL : http://journals.openedition.org/germanica/3184 ; DOI : https://doi.org/ 10.4000/germanica.3184 


\section{Le roman policier entre passé et présent}





\title{
Die Kriminalromane von Wolf Haas. Ein kritischer Dialog mit Tradition und Gegenwart
}

\author{
Valentina SERRA \\ Università degli Studi di Cagliari
}

\begin{abstract}
„Es ist das Spiel mit dem Interesse, das mich vor allem interessiert. Wie weit kann ich den Leser noch ärgern, ab wann muss ich ihm wieder entgegenkommen?"1
\end{abstract}

\section{Prämisse}

Eine derzeit umfangreiche kritische Literatur betont auf der Grundlage ernsthafter und akribischer Forschungen die extreme Neuheit des Schaffensprozesses des österreichischen Schriftstellers Wolf Haas (1960), vor allem in Bezug auf die erfolgreichen Kriminalromane über den Privatdetektiv Simon Brenner ${ }^{2}$ und auf die geniale Intuition eines „göttlich allwissend[en] und stammtischpolternd[en]“ Erzählers ${ }^{3}$, der

1. - Wolf Haas in Susanne Rössler, ,Ich spinne so vor mich hin, und irgendwann ist das Buch fertig“, in: Volltext. Zeitung für Literatur, 2 (2002), S. 2.

2. - Dabei handelt es sich um die Bücher Auferstehung der Toten (1996), Der Knochenmann (1997), Komm, süßer Tod (1998), Silentium! (1999), Wie die Tiere (2001), Das ewige Leben (2003), Der Brenner und der liebe Gott (2009), Brennerova (2014).

3. - Thomas Klingenmaier, „Der Brenner, also praktisch Dings“, in: Sonntag 
„die klare Unterscheidbarkeit zwischen homo-, hetero- und autodiegetischem Erzählen verneint“4.

Kritiker und Literaturwissenschaftler haben bisher die Beziehungen zwischen den Werken von Haas und der Tradition anhand der Erzählstrategien und der Zuordnung der Gattung des Kriminalromans im Detail untersucht ${ }^{5}$. Sie haben vor allem einhellig hervorgehoben, dass seine Romane in erster Linie nie die Handlung ${ }^{6}$ sondern die „Halbsätze, Wortauslassungen, mitten im Schreiben den Kurs wechselnde[r] Gedanke[n]"7 betonen.

Ziel des vorliegenden Beitrags ist, den Faden dieses Diskurses im Lichte seines Bezugs zur literarischen Tradition thematisch wiederaufzunehmen, der oft vom Autor selbst widerlegt wird.

Ohne jeglichen Anspruch auf Vollständigkeit wird hier versucht, einige mit dem Thema des Spiels verbundene Elemente hervorzuheben, in Richtung einer Nacharbeit „klassischer“, in dieser Gattung tief verwurzelter Kernthemen, wie etwa die unscharfen Gegensätze zwischen Gut und Böse, (Selbst)-Justiz und Schuld.

\section{Wolf Haas: der Krimiautor, der keinen Krimi mag. Auseinandersetzung mit der literarischen Tradition}

Der Überbegriff „Kriminalroman“ bezeichnet strukturgleiche Romane, die verschiedene Untergruppierungsmöglichkeiten seitens eines bestimmten Falles und bestimmter Lösungen anbieten. Wenn die Lösung eines Falles vom rationalen Kalkül eines Ermittlers dominiert erscheint, handelt es sich um eine Detektivgeschichte ${ }^{8}$. Ihre Ursprünge und Merkmale, die in ihrer logischen Argumentation auf den aufklärerischen Rationalismus und, im Fall vom Mystery und Horror, auf

aktuell, 16.9.2001; vgl. dazu auch Peter Mußler (a), Hauptdarsteller Hilfsausdruck. Wolf Haas' Brenner-Romane - Wenn der Erzähler seinen Figuren die Show stiehlt, Hamburg, Disserta Verlag, 2015.

4. - Peter Mußler (b), Der Brenner und die Narratologie. Eine erzähltheoretische Untersuchung von Wolf Haas' Krimireihe, München, GRIN Verlag, 2015.

5. - Vgl. Hannah Scharf, Wolf Haas und der Kriminalroman: Unterhaltung zwischen traditionellen Genrestrukturen und Innovation, Hamburg, Diplomica Verlag, 2014.

6. - Vgl. Richard Reichensperger, ,Der abschweifende Kinderblick im Krimi Wolf-Haas-Tage beim ,steirischen herbst'“, in: Der Standard, Wien, 21.10.2003 und Günther Stocker, ,Zwischen Zell am See und Teneriffa - Neue Krimis von Wolf Haas und Günter Brödl“", Literatur und Kritik, 32 (1997), S. 82.

7. - Klingenmaier, a.a.O.

8. - Walter Seifert, Friedrich Dürrenmatt. Der Richter und sein Henker, München, Oldenbourg, 1975, S. 12. 
die romantische Gothik zurückgreifen, sind, wie eine umfangreiche Bibliographie zeigt, genau untersucht worden 9 .

Wie bekannt, wurde das rationale Element, das allmählich zur Lösung eines Rätsels führt, in der ersten Hälfte des neunzehnten Jahrhunderts von Edgar Allan Poe (1809-1849)10 eingeführt mit der sogennanten Detection, d. h. der vom Detektiv verwendete Prozess, eine Wahrheit zu enthüllen, der durch die Anwendung eines fiktiven, erkenntnistheoretischen und szientistischen Ansatzes zur Realität erfolgt.

Für lange Zeit an den Rand der sogenannten „Hochliteratur“ verbannt, begann das Genre zwischen den zwanziger und dreißiger Jahren des letzten Jahrhunderts seine literarische Würde zu gewinnen und seine unzähligen Möglichkeiten in der analytischen Darstellung der Gesellschaft und in der psychologischen Introspektion der Charaktere zu zeigen ${ }^{11}$.

In den zwanziger Jahren des letzten Jahrhunderts eroberten Schriftsteller wie Dashiell Hammett (1894-1961) und Raymond Chandler (1888-1959) die Aufmerksamkeit des breiten Publikums durch die sogenannten hard-boiled-Romane ${ }^{12}$, die die groteske und gewaltige Welt der amerikanischen Metropolen darstellen.

In Frankreich wird gleichzeitig der ,psychologische' Roman von Georges Simenon (1903-1989) mit seinem aufrechten und den Verbrechern von Hammett und Chandler gegenübergestellten Kommissar Maigret sehr berühmt.

9. - Vgl. u. a. Tzvetan Todorov, Typologie du roman policier, in Poétique de la prose, Paris, Editions du Seuil, 1971, S. 55-65; Thomas Narcejac, Une machine à lire: le roman policier, Parigi, Denoël/Gonthier, 1975; Edgar Marsh, Die Kriminalerzählung. Theorie - Geschichte - Analyse, München, Winkler, 1983; Umberto Eco - Thomas Albert Sebeok (Hrsg.), The Sign of Three. Dupin, Holmes, Peirce, Bloomington, Indiana University Press, 1983; Yves Reuter, Le roman policier, Paris, Éditions Nathan, 1997; Peter Nusser, Der Kriminalroman, Stuttgart, Metzler, 2003.

10. - Poe war auch der erste Theoretiker dieses Genres, da er in The Philosophy of Composition (1846) sein Kompositionsverfahren als bewußtes intellektuelles Arbeitsprogramm erläuterte.

11. - Auch im deutschsprachigen Raum haben sich berühmte Schriftsteller und Philosophen, wie etwa Siegfried Kracauer (Der Detektiv-Roman. Ein philosophischer Traktat, 1925), Ernst Bloch (Philosophische Ansicht des Detektivromans, 1965) und Bertolt Brecht (Über die Popularität des Kriminalromans, 1938) damit beschäftigt, die Mechanismen des Detektivromans im epistemologischen, soziologischen und literarischen Sinn zu untersuchen. Ernst Bloch fand in der Ödipus-Geschichte der „Urstoff des Detektorischen schlechthin“ (in: Jochen Vogt (Hrsg.), Der Kriminalroman. Poetik - Theorie - Geschichte, München, Fink, 1998, S. 38-51, hier S. 46) während die Reflexion Bertolt Brechts als einer der ersten Versuche gilt, den Wert dieser Gattung objektiv einzuschätzen.

12. - Peter Nusser (a.a.O., S. 48) ordnet den amerikanischen hard-boiled-Krimi, den Heftromankrimi und den Spionageroman dem Thriller zu. 
Das Werk dieser Schriftsteller trägt dazu bei, die Figur eines unbesiegbaren, der deduktiven und rationellen Methode verpflichteten Ermittlers zu entmythisieren und den neuen Detektiv als einen normalen, alltäglichen Helden zu schaffen.

Der endgültige Tod des traditionellen Detektivromans und des unfehlbaren Detektivs wurde von Friedrich Dürrenmatt mit dem Werk Das Versprechen. Requiem auf den Kriminalroman (1958) dargestellt. Dieses Werk bezeugt den Untergang des logischen Positivismus, der Gewissheit eines rationalen Prinzips, das die Welt meistert und erlaubt, das menschliche Handeln vorherzusagen. Durch die Einfügung des Zufalls, der den Mörder hinrichtet, zerstört Dürrenmatt die Möglichkeit jedes logischen und programmatischen Handelns des selbstbewussten Ermittlers.

Der Kriminalroman zählt heute nicht mehr zur Kategorie der Trivial- oder Verbraucherliteratur, sondern wird gerade für seine Fähigkeit geschätzt, vorgefasste Dichotomien und starre Taxonomien zu hinterfragen ${ }^{13}$. Er wird vor allem als kritische Repräsentationsform der zeitgenössischen Wirklichkeit und Denunziation ihrer Verzerrungen gepriesen: heutzutage stellt der Kriminalfall keine Zerstörung eines gegebenen Gleichgewichts dar, sondern enthüllt eine ständige, schmachvolle, gesellschaftliche Ungerechtigkeit.

Das Werk des Schriftstellers Wolf Haas stellt eine interessante und aktuelle Variation dieses Genres dar, welche Unterhaltung und kritische Reflexion über die typischen Verzerrungen der österreichischen Gesellschaft glücklich vereint, anhand eines poetologischen und stilistischen Experimentierens und einer kreativen und nicht immer offensichtlichen Wiederbelebung der literarischen Tradition.

Die Romane dieses Autors wurden abwechselnd als Detektiv- bzw. Kriminalromane oder Thriller bezeichnet ${ }^{14}$, obwohl sein literarisches Werk jeden Versuch widerlegt, es in festen Kategorien zu fassen ${ }^{15}$.

Die Brenner-Serie trägt eigentlich noch einige Spuren des Detektivromans, da die Architektur der traditionellen Detection und die literarische Tradition einen klaren Einfluss auf die narratologische und thematische Struktur ausüben ${ }^{16}$, zeigt aber auch bestimmte kreative Merkmale im Stil und in der Konstruktion der Figuren.

13. - Siehe dazu Reuter, a.a.O. und Günter Waldmann, Theorie und Didaktik der Trivialliteratur, München, Fink, 1973.

14. - Siehe Richard Reichensperger, „Der abschweifende Kinderblick im Krimi Wolf-Haas-Tage beim ,steirischen herbst'“, in: Der Standard, Wien, 21.10.2003, S. 26.

15. - Franz Schuh, „Und jetzt so was“. Überlegungen zum literarischen Rang von Wolf Haas, in: Schreibkraft. Brennermania, 9 (2003), S. 14-25.

16. - Vgl. Richard Austin Freeman, „The Art of the Detection Story“, in Nineteenth Century and After, 95 (1924), S. 714-715. 
Der Autor beherrscht diese Gattung mit großer Sicherheit und, obwohl er behauptet, er lese keine Kriminalromane ${ }^{17}$ und bewundere nur das Werk von Chandler und Hammett ${ }^{18}$, ohne einen direkten Einfluss von der Tradition auf sich zu spüren, verraten seine Werke eine verwandtschaftliche Verbindung mit dem angloamerikanischen Detektivroman, dem Noir und der deutschsprachigen Tradition. Die humorvollen Aussprüche, die an Georges Simenon erinnern und die bereits typisch Haas'schen Witze und sein Sarkasmus nähern sich der akribischen Beschreibung blutiger Ereignisse der Hard Boiled Romane ${ }^{19}$.

Der von der Erfahrung als Werbetexter beeinflußte Schreibprozess bei Haas basiert auf einer anspruchsvollen Verwendung von Dialekt, Neologismen und Wortspielen und erzielt ein eigentümliches linguistisches Spiel mit einer gekonnt konstruierten Sprache, so dass seine Romane manchmal als „,mutig“ bezeichnet werden ${ }^{20}$.

\section{Die „österreichischen" Krimis von Wolf Haas}

Das reiche literarische Schaffen Österreichs hat eine lebhafte Debatte über die Existenz eines typisch österreichischen Kriminalromans ausgelöst, der besondere Merkmale tragen soll ${ }^{21}$.

Ein Argument für eine typisch österreichische Ausbildung des Genres sind die strukturellen, stilistischen und thematischen Gemeinsamkeiten zwischen verschiedenen Werken, die einen angelsächsischen Einfluß zeigen, wie z. B. die klassischen Schemata des sogenannten Whodunit, der auf Edgar Allan Poe und Arthur Conan Doyle zurückgeht. Diese Merkmale werden aber in den sozio-kulturellen österreichischen Kontext transportiert, vor allem im Fall des sogenannten Regionalkrimis, einer Gattung, die auch in der deutschen Literatur viel Erfolg hat ${ }^{22}$. Ortspiele

17. - Vgl. René Freund, „Der Wolf im Haaspelz - Krimi-Autor Wolf Haas mag keine Kriminalromane“, in Wiener Zeitung, Wien, 22.12.2000, S. 10 (Beilage).

18. - Vgl. Christian Seiler, „Gehört gehört. Der Werbetexter Wolf Haas und seine neue, leidenschaftliche Karriere als Kriminalschriftsteller“, in Profil, 30.6.1997, S.112113 und Ilse Aichinger, ,'Wie schreiben Sie eigentlich?', 'Pubertär'“, in: Der Standard, 20.07.2001, S. 25.

19. - Siehe Renate Peschik-Vodosek, Von der Auferstehung zum ewigen Leben: Die Brenner-Krimis von Wolf Haas im Gattungskontext, Diplomarbeit, Universität Wien, 2008, S. $48 \mathrm{ff}$.

20. - Froschauer, a.a.O., S. 7. Über die Sprache und den Stil von Haas siehe u.a. Sigrid Nindl, Wolf Haas und sein kriminalliterarisches Sprachexperiment, Berlin, Erich Schmidt Verlag, 2010 und Mußler (a) und (b), a.a.O.

21. - Vgl. Peschik-Vodosek, a.a.O., S. 42 und Friedbert Aspetsberger (Hrsg.), Nichts Neues - Trends und Motive in der (österreichischen) Gegenwartsliteratur, Innsbruck, StudienVerlag, 2003.

22. - Sandro Moraldo (Hrsg.), Mord als kreativer Prozess. Zum Kriminalroman der Gegenwart in Deutschland, Österreich und der Schweiz, Heidelberg, Universitätsverlag Winter, 2005. 
und Wortspiele, Schauplätze und Sprache sollten also den typisch österreichischen Kriminalroman kennzeichnen ${ }^{23}$.

Manche Wissenschaftler haben mehrere Unterkategorien des österreichischen Krimis identifiziert, vom ,Wiener Krimi' - wo die Hauptstadt ,als Zentrum einer autoritativen, total verbürokratisierten, unzugänglichen, nachgerade kafkaesken Macht" 24 dargestellt wird,zum ,Regionalkrimi', vom ,gesellschaftskritischen Soziokrimi' zum ,politischen Krimi', vom ,Frauenkrimi' bis zum ,satirischen Krimi' und ,psychoanalytischen Krimi'25.

Franz Schuh hat bereits die gesellschaftskritische Funktion beschrieben, die viele österreichische Krimischriftsteller (darunter Wolf Haas, Eva Rossmann, Kurt Bracharz, Jürgen Benvenuti und Ernst Hinterberger) von der Avantgarde der siebziger Jahre übernommen hätten ${ }^{26}$. In diesem Sinn wird der Kriminalroman als Form einer AntiHeimat-Literatur interpretiert ${ }^{27}$, die eine Gesellschaft der Devianzen und Kriminalfälle kritisch porträtiert ${ }^{28}$.

Die Romane von Haas wurden sowohl als Wiener- als auch Regionalund Soziokrimis bezeichnet. Im allgemeinen kann man sagen, dass das Werk des Autors den heutigen österreichischen Krimi in fast allen seinen Varianten ausgezeichnet darstellt, wobei die Untergattung des Soziokrimis, der die Devianzen der österreichischen Gesellschaft betont, die wichtigste Rolle spielt.

Im Haas'schen Schaffen stellt der Kriminalfall keine äußerliche Gefahr dar, die das soziale, interne Gleichgewicht gefährdet, sondern er wird zur Enthüllungsmöglichkeit verschiedener gesellschaftlichen Übel. Die Brenner-Romane spiegeln ein von der Provinz bis zur Wiener Hauptstadt typisch österreichisches Milieu wider, mit dem ironischen Stil

23. - Siehe Arno Russegger, „Ortspiele. Wortspiele. Aspekte kriminalistischen Erzählens in der österreichischen Gegenwartsliteratur", in: Sandro Moraldo, a.a.O., S. 76.

24. - Russegger, a.a.O., S. $78 \mathrm{ff}$.

25. - Siehe Nusser, a.a.O., S. $107 \mathrm{ff}$.

26. - Vgl. Franz Schuh, „Hinein in den Kanon mit ihm! Plädoyer für das Niedrige und das Erhabene, für den Kulturkampf und für Wolf Haas“, in: Kolik. Zeitschrift für Literatur, 18 (2002) S. 19ff.

27. - Siehe Kateřina Hrůzová, Österreich als „Mordschauplatz“. Wolf Haas' Kriminalromane im Kontext der Anti-Heimatliteratur, Diplomarbeit, Südböhmische Universität in České Budějovice, 2011.

28. - „Dabei wird das Wertsystem der österreichischen Gesellschaft einer hochnotpeinlichen Analyse unterzogen, kollektive und individuelle Vorstellungen werden, was denn unter Normalität oder Sitte und Moral überhaupt zu verstehen sei, einander gegenübergestellt. In der österreichischen Kriminalliteratur führt das - im Großen und Ganzen - zur Gestaltung eines zynisch eingefärbten Weltbilds, welches aus einem kalkulierten Wechselspiel von tabusinnigen und tabubrecherischen [...] resultiert", Russegger, a.a.O., S. 76 ff. 
einer alltäglichen, mundartlichen Sprache (der sogenannten fingierten Mündlichkeit) und einer der Konkreten Poesie ähnelnden anti-intellektualistischen Stellung, die er während seines Germanistikstudiums erforscht hat ${ }^{29}$.

Diese teilweise zynische Wirklichkeitsdarstellung ähnelt den von Friedrich Dürrenmatt meisterhaft entwickelten Stilformen und Themen. Ein erwähnenswertes Beispiel dafür sind die Ähnlichkeiten zwischen Dürrenmatts Besuch der alten Dame und dem ersten Brenner-Roman Auferstehung der Toten, wo sich eine Frau an einem pensionierten amerikanischen Ehepaar rächt, das ihr in der Vergangenheit den von einer inzestuösen Beziehung geborenen Sohn weggenommen hatte.

\section{Simon Brenner und seine Vorgänger}

Als müder, schlapper und träger Anti-Held ${ }^{30}$, der keinem ideellen Justizprinzip anhängt, sondern die Frühpension anstrebt, zerstört Brenner die Figur des traditionellen Detektivs. Mit seinen Schwächen, seinem Zynismus und seiner Lebensenttäuschung zweifelt er, im Gegensatz zu Gilbert K. Chesterton's Father Brown, an der Möglichkeit, durch sein Handeln eine beliebige moralische oder gesellschaftliche Ordnung wiederherzustellen.

Brenner verfügt aber über ein außergewöhnliches Gehör und ein Unbewusstes, das ihn zur Lösung der Fälle führt; er stützt sich also mehr auf Intuition als auf Rationalität und benutzt keine induktive oder deduktive Methode, sondern eine assoziative und intuitive: „Es“ und Logik führen ihn pünktlich - oder besser mit einer gewissen Verspätung - zum Mörder ${ }^{31}$.

Diese ,kreative' Methode, die auf die Beobachtung von Kleinigkeiten und Nebensächlichkeiten beruht, erweist sich als Zitat von Holmes' und Poirots Ermittlungsprozess, so wie Brenners Wohnungslosigkeit, die als Merkmal des Detektivs gekennzeichnet wird ${ }^{32}$.

29. - Haas promovierte mit einer Dissertation mit dem Titel Sprachtheoretische Grundlagen der konkreten Poesie (Stuttgart, Heinz, 1990) und wird in seinem linguistischen und stilistischen Experimentieren von der Bewegung der Konkreten Poesie, vom Werk Ernst Jandls und der italienisch-französischen Schule der OuLiPo beeinflußt (siehe Nindl a.a.O. und Astrid Poier-Bernhard, Viel Spaß mit Haas! - oder ohne Haas, je nachdem, wie du das jetzt sehen willst, Wien, Sonderzahl, 2003).

30. - Siehe Froschauer, a.a.O., S. 17; Volker Albers, „Wolf Haas: ,...weil ich so ein Weichei bin'“, in: Hamburger Abendblatt, 01.03.2005, S. 7 und Andrea Praß1, Wolf Haas: Schreibtischtäter und ,Dudendetektiv', Diplomarbeit, Graz, 2001, S. 9.

31. - Siehe Astrid Poier-Bernhard, „Wörter auf großer Flamme. Wolf Haas als Sprachspieler“, in: Schreibkraft. Brennermania, 9 (2003), S. 4-13, Peter Mußler (a), a.a.O.

32. - Vgl. Peschik-Vodosek, a.a.O., S. 56 ff. 
Der Autor selbst exemplifiziert den Prozess der Detection durch ein indirektes Zitat des Meisters Edgar Allan Poe ${ }^{33}$, der behauptete, dass die Wahrheit nicht immer in einem Brunnen, sondern manchmal in der Oberfläche steckt, wo wir sie nicht suchen ${ }^{34}$. Im Roman Silentium! philosophiert Brenner über seinen sinnlosen Ermittlunsprozess anhand der Metapher von Plastiktaschen, die nichts als andere Plastiktaschen enthalten:

Man öffnet eine Tasche in der Hoffnung, daß etwas drinnen ist, aber es ist wieder nur eine Tasche drinnen, und wenn man die aufmacht, ist wieder nur eine Tasche drinnen, und ewig so fort. [...] Ich meine nur, daß man im nachhinein immer drauf kommt, daß man an der falschen Stelle gesucht hat. Man sucht Geheimnisse, obwohl es offen zutage liegt. Die wichtigen Dinge sind nicht versteckt wie die Sachen in einer Tasche, sondern ganz offenkundig (S. 191 ff.).

Simon Brenner könnte schließlich als Hommage auf Dürrenmatts Bärlach (Der Richter und sein Henker, 1951) und Matthäi (Das Versprechen) gelesen werden, als menschlicher, schwacher und manchmal lächerlicher Ermittler, der die Niederlage eines szientistischen ,dupinschen' Verfahrens darstellt ${ }^{35}$.

Brenner widerlegt das Klischee des genialen Detektivs wie Sherlock Holmes oder Auguste Dupin, die in der Lage sind, die Leser mit ihrer fast übermenschlichen Genialität zu überraschen. Dem Haas'schen Hauptcharakter fehlen außerdem die spekulative Fähigkeit und der Abstand zum gesellschaftlichen Milieu, die die Protagonisten des Detektivromans kennzeichnen, die physische Stärke und Entschlossenheit der Thrillerhelden und der für die hard-boiledRomane typische Wunsch nach sozialer Erlösung.

\section{Spiel und Tradition, Spiel mit der Tradition}

„Es ist das Spiel mit dem Interesse, das mich vor allem interessiert. Wie weit kann ich den Leser noch ärgern, ab wann muss ich ihm wieder entgegenkommen?"36. Diese Aussage von Wolf Haas verweist auf die

33. - Vgl. Alida Bremer, Kriminalistische Dekonstruktion. Zur Poetik der postmodernen Kriminalromane, Würzburg, Königshausen und Neumann, 1999 und Peschik-Vodosek, a.a.O., S. 70.

34. - Edgar Allan Poe, Die Morde in der Rue Morgue (The Murders in the Rue Morgue), in Detektivgeschichten, übers. von Hans Wollschläger, München, DTV, 1998, S.5-50, hier S. 25.

35. - Für eine solche Interpretation bei Dürrenmatt siehe Francesco Fiorentino, „La letteratura svizzera di lingua tedesca del Secondo Novecento”, in: Marino Freschi (Hrsg.), Storia della civiltà letteraria tedesca, Torino, UTET, 1998, Bd. 2, S. 618-844.

36. - Wolf Haas in Rössler, a.a.O. 
Bedeutung des Spiels in seinem Werk, das sich auch thematisch mit der literarischen Tradition verbindet.

Der Mechanismus des Detektivromans wurde bereits von S. S. Van Dine (1887-1939) mit "einem sportlichen Wettkampf zwischen dem Detektiv und dem Leser, ${ }^{37}$, mit seinen Regeln und Fairplay verglichen. Die Figuren im Thriller werden in zwei Gruppen eingeteilt, die eine auf der Seite des Ermittlers (Helfer) die andere gegen ihn, also die Helfer des Täters ${ }^{38}$. Diese manichäische Opposition ähnelt der Regel eines Spiels, das im Werk von Haas eine besondere Rolle spielt.

Das Thema des Spiels findet wie bekannt bei Friedrich Dürrenmatt eines der bedeutendsten und verfeinertsten Beispiele, die die wesentlichen ontologischen und existenziellen Fragen der menschlichen Gesellschaft darstellen ${ }^{39}$. Bedeutungsvoll sind in diesem Sinn die Metaphern aus dem Bereich des Sports, das Billardspiel (Justiz, 1985)40 oder die Rollenspiele (wie etwa im Monstervortrag über Gerechtigkeit und Recht 1969) ${ }^{41}$.

Am häufigsten tritt aber im Werk von Dürrenmatt das Schachspiel auf, da der schweizerische Dramaturg an dem Duellcharakter des Spiels als „,idealisierte[n] Kampf“42 interessiert war. „Die Konfrontation zweier Spieler, die bei prinzipiell gleichen Chancen einander gegenübersitzen" $" 33$ habe Dürrenmatt fasziniert, wobei die Gegner ein gewisses „logisches Wagnis“44, also eine Kombination von „Taktik, Strategie, kühle Berechnung und Intuition“45 haben sollen. Das Spiel

37. - S. S. Van Dine (alias Willard Huntington Wright), „Zwanzig Regeln für das Schreiben von Detektivgeschichten“ (1946), in: Jochen Vogt (Hrsg.), Der Kriminalroman. Zur Theorie und Geschichte einer Gattung, München, Willhelm Fink Verlag, 1971, Band 1, S. 143-147, hier S. 143.

38. - Vgl. Nusser, a.a.O., S. $54 \mathrm{ff}$.

39. - Vgl. dazu Bernhard Auge, Friedrich Dürrenmatts Roman ,Justiz'. Entstehungsgeschichte, Problemanalyse, Einordnung ins Gesamtwerk, Münster, LIT Verlag, 2004, S. $323 \mathrm{ff}$. 1991.

40. - Friedrich Dürrenmatt, Justiz, in Gesammelte Werke IV, Zürich, Diogenes,

41. - Ebd., Monstervortrag über Gerechtigkeit und Recht, in: Gesammelte Werke VII , a.a.O., S. $628 \mathrm{ff}$.

42. - Hans-Dietrich Irmscher, „Das Schachspiel als Metapher. Bemerkungen zum ,komödiantischen Denken' Friedrich Dürrenmatts“, in: Hans-Dietrich Irmscher - Werner Keller (Hrsg.), Drama und Theater im 20. Jahrhundert. Festschrift für Walter Hinck, Göttingen, Vandenhoeck \& Ruprecht, 1983, S. 333-348, hier S. 336. Dürrenmatt hatte auch vor, einen Roman mit dem Titel Die Schachspieler zu veröffentlichen (Bernhard Auge, a.a.O., S. 323).

43. - Irmscher: a.a.O., S. 336; siehe auch Friedrich Dürrenmatt, Albert Einstein. Ein Vortrag, in: Gesammelte Werke VII, a.a.O., S. 733-766.

44. - Dürrenmatt, Albert Einstein, a.a.O., S. 746.

45. - Ebd., S. 745. 
sei für den Schrifsteller vor allem eine Darstellung der literarischen Metapher selbst, die die Wirklichkeit nachahmt.

Das Schachspiel taucht in bedeutungsvoller Weise in Der Richter und sein Henker ${ }^{46}$ auf, wo der Kampf zwischen Bärlach (der von Dürrenmatt am Ende des Romans als „unerbittlicher Schachspieler“ bezeichnet wird, S. 113) und Gastmann ,eine Art Schachpartie ist, in der Menschen die Figuren darstellen“47.

Im Verdacht (1953) ${ }^{48}$ erscheint das Schachspiel als Metapher eines manichäischen Widerspruchs zwischen dem Guten (im Bett liegenden Bärlach) und dem Bösen (Emmenberger), wobei die Logik und der Wille der Spieler durch den Zufall zugrunde gehen, der im Werk Dürrenmatts jeden Versuch des Menschen scheitern lässt, die Welt zu meistern.

Ähnlich wie die Schachpartie, fungiert das Billardspiel bei Dürrenmatt als metaphorisches Mittel, das das Weltgeschehen den Lesern begreifbar macht ${ }^{49}$. In Justiz spiegelt das Billard die Existenz der als Kugeln dargestellten Charaktere wider, deren Weiterleben oder Tod von einem fürchterlichen, Gott ähnelnden Mörder bestimmt wird: ,[Gott] spielt Billard. Er gibt den ersten Stoß und die Welt setzt sich in Bewegung!“50.

Im Werk findet aber kein Duell statt, da Kohler sein Spiel allein treibt, um eine allgewaltige wirtschaftliche Macht zu erobern. Das Spiel enthüllt sich hier nochmals ,als Modell der Wirklichkeit [...]. Als eine ihrer möglichen Vereinfachungen" 51 .

Wolf Haas nimmt diese Metaphern wieder auf, auch wenn er dem Zufall keine besondere oder präzise Rolle übergibt (der Zufall taucht in seinem Werk nur als zufällige Hilfe Brenners in der Lösung der Fälle auf) $)^{52}$.

In Silentium!, einer der wichtigsten Romane der Brenner-Serie, erhält der Tischfußball eine hervorragende materielle und symbolische Geltung: im Inneren eines Tischfußballtisches eines katholischen Internats wird die zerstückelte Leiche eines ehemaligen Schülers aufge-

46. - Ebd., Der Richter und sein Henker, in: Gesammelte Werke IV, a.a.O.

47. - Auge, a.a.O., S. 325.

48. - Friedrich Dürrenmatt, Der Verdacht, in: Gesammelte Werke IV, Zürich, Diogenes, 1991.

49. - Auge, a.a.O., S. 329.

50. - Friedrich Dürrenmatt, „Filmerzählung“ zu Justiz, Reinschrift 140, S. 106 zit. nach Auge, a.a.O., S. 332.

51. - Ebd., Justiz, a.a.O., S. 652; vgl. auch Auge, a.a.O., S. 332.

52. - Ulrich Schulz-Buschhaus meint, dass die Aufklärung als Produkt des Zufalls ein Merkmal des neuen deutschen Kriminalromans ist; Vgl. Ulrich SchulzBuschhaus, „Die Ohnmacht des Detektivs. Literarhistorische Bemerkungen zum neuen deutschen Kriminalroman“, in: Karl Ermert - Wolfgang Gast (Hrsg.), Der neue deutsche Kriminalroman - Beiträge zu Darstellung, Interpretation und Kritik eines populären Genres, Evangelische Akademie Loccum, Rehburg-Loccum, 1985, S.10-18. 
funden und das Spiel selbst wird zur Metapher des Kampfes Brenners gegen den über jeden Verdacht erhabenen Mörder.

Die materielle und symbolische Bedeutung des Spiels wird im Buch mehrfach angedeutet. Während er eine Partie mit sich selbst spielt, (obwohl der Tischfußball „kein schönes Spiel für einen allein“ sei, S.181) enthüllt Simon Brenner den Mörder und die Begebenheiten, die ihn zum Verbrechen geführt haben. Für den Privatdetektiv wird der Tischfußball ,die beste Nachdenkhilfe [...], die es auf der Welt überhaupt gibt“" (Ebd.): seine Gedanken ähneln dem Ball, der hin und her geschossen wird und endlich zum Tor kommt, ,weil solange die Kugel gerollt ist, sind auch die Gedanken vom Brenner ein bißchen herumgerollt" (S. 184).

Der Tischfußball gleicht im erweiterten Sinn der Lage des hilflosen Protagonisten und der anderen Romanfiguren, die für lange Zeit vom Mörder herummanövriert wurden: ,und ein bißchen ist er sich jetzt selber wie so ein Tischfußballmännchen vorgekommen. Und der René ein Tischfußballmännchen, und die Dr. Ogusake ein Tischfußballmännchen, und der Gottlieb ein Tischfußballmännchen, und der Waldbrand ein Tischfußballmännchen, und alle anderen auch nur Tischfußballmännchen, mit denen der Präfekt Fitz gespielt hat"“ (S. 181 f.).

Die manichäische Opposition des lebenswichtigen Spiels wird vom Tischfußballtisch symbolisiert, wobei Brenner mit der blauen (himmlischen) gegen die rote (höllische) Mannschaft spielt. Die beschränkten Bewegungen der Tischfußballmännchen (,So wie man beim Tischfußball auch nur alle Männchen an einer Stange bewegen kann oder keines“, S. 189) gleichen seinem sinnlosen Herumlaufen als er vergebens versucht, den Mörder, die Ermordungen zu stoppen: „die roten Männer sind herrenlos in der Gegend herumgestanden. Nur wenn der Brenner einen von ihnen angeschossen hat, haben sie sich sinnlos in der Luft gedreht wie ein Detektiv, der keinen Plan hat und nur hin und wieder von einem Denkstoß gebeutelt wird“" (S. 182).

Im Werk Dürrenmatts wird das Spiel sowohl aus einer Außen- (die Spieler, die die Figuren bewegen) als auch aus einer Innenperspektive (die Menschen, mit denen gespielt wird) dargestellt. Auch im Roman Silentium! wird diese doppelte Perspektive deutlich, da die Außenperspektive vom fiktiven Erzähler dargestellt wird, während die Innenperspektive der Hilflosigkeit und Verlegenheit bei Brenners Alleinspielen eine erfolgreiche Darstellung erzielt.

Die Spieler von Haas sind also wie die von Dürrenmatt meisterhaft beschriebenen Schachfiguren, die ,innerhalb der Partie [sind], für sie stellt sich das Spiel ganz anders dar, sie schlagen Figuren und werden von Figuren geschlagen, sie sind in eine unbarmherzige Schlacht 
verwickelt, sie können nichts vom Schlachtplan wissen, der sie lenkt, wenn es ihn überhaupt gibt" 53 .

Das Thema des Spiels ist bei beiden Autoren eng mit dem Konzept von Schuld verbunden. Das Werk von Haas steht in dieser Hinsicht der Tradition Dürrenmatts nahe, da er eine wirklichkeitsgetreue Welt darstellt, wo mehrere Charaktere sich als schuldig zu erkennen geben.

Die Legitimität einer Selbstjustiz ist in diesem Sinn eine der Lösungen, die beide Schriftsteller mit der Darstellung einer korrupten, widersprüchlichen und den ökonomischen, religiösen und politischen Interessen einer Minderheit unterlegenen Gesellschaft vorschlagen. Wie Bärlach seinen Feind von seinem Helfer töten läßt, so erlaubt Brenner seinem Freund René, den Mörder zu ersticken, wobei die traditionelle Rolle von Opfer und Täter, Guten und Bösen provokativ problematisiert wird. Das literarische Schaffen von Wolf Haas bestätigt also die traditionell der „Hochliteratur“ zugewiesenen Rolle der kritischen Darstellung der Gegenwart, eine Rolle die im Werk Friedrich Dürrenmatts eines der hervorragendsten „klassischen“ Beispiele findet. Allen Behauptungen der Kritiker und des Schriftstellers selbst zum Trotz, werden auch die thematischen Aspekte dieser Romane von der großen literarischen Tradition der Kriminalromane beeinflusst. 